Supporting Information for

\title{
Monolithic Full-Stokes Near-Infrared Polarimetry with Chiral Plasmonic Metasurface Integrated Graphene- Silicon Photodetector
}

Lingfei Li, ${ }^{1,2}$ Junzhuan Wang,,$^{*}$ Lei Kang, ${ }^{3}$ Wei Liu, ${ }^{1}$ Li Yu, ${ }^{2}$ Binjie Zheng, ${ }^{2}$ Mark L. Brongersma, ${ }^{4}$ Douglas H. Werner, ${ }^{3}$ Shoufeng Lan, ${ }^{5}$ Yi Shi, ${ }^{2}$ Yang Xu,${ }^{1 *}$ and Xiaomu Wang $^{2 *}$

${ }^{1}$ School of Micro-Nanoelectronics, ZJU-Hangzhou Global Scientific and Technological Innovation Center, ZJU-UIUC Institute, State Key Labs of Silicon Materials and Modern Optical Instrumentation, Zhejiang University, Hangzhou 311200, China

${ }^{2}$ School of Electronic Science and Engineering, Nanjing University, Nanjing 210093, China

${ }^{3}$ Department of Electrical Engineering, The Pennsylvania State University, University Park, Pennsylvania 16802, United States

${ }^{4}$ Geballe Laboratory of Advanced Materials, Stanford University, Stanford, California 94305, United States 
${ }^{5}$ Department of Mechanical Engineering, Texas A\&M University, College Station, Texas 77843, United States

*Corresponding authors: xiaomu.wang@nju.edu.cn, yangxu-isee@zju.edu.cn and wangjz@nju.edu.cn 


\section{Supplementary Figures}

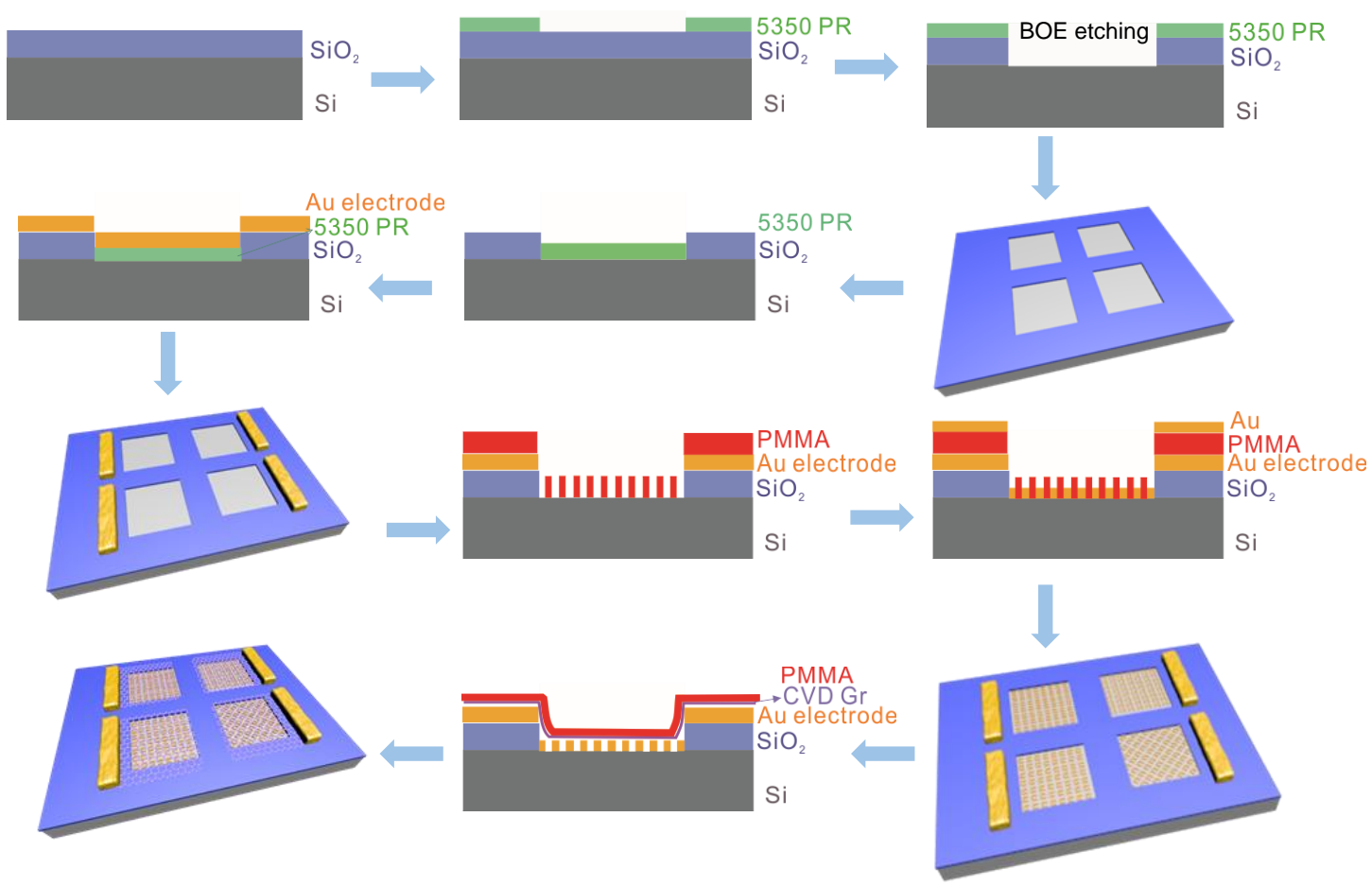

Figure S1. Schematic of fabrication processes for the four-pixel metasurface polarimeter. 5350 photoresist (PR) is used for ultra-violet lithography to pattern the silicon window and electrodes. $\mathrm{Au}(37 \mathrm{~nm}) / \mathrm{Cr}(3 \mathrm{~nm})$ plasmonic nanostructures are fabricated by high-resolution electron-beam lithography and evaporation, followed by lift-off processes. 
(a)

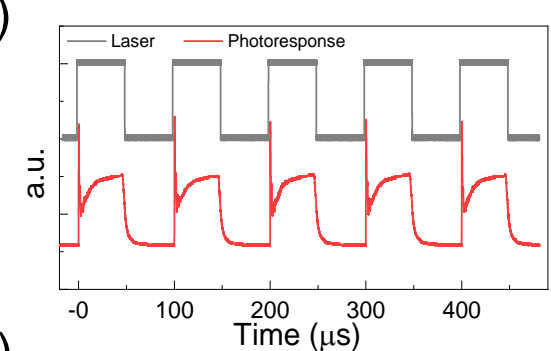

(b)

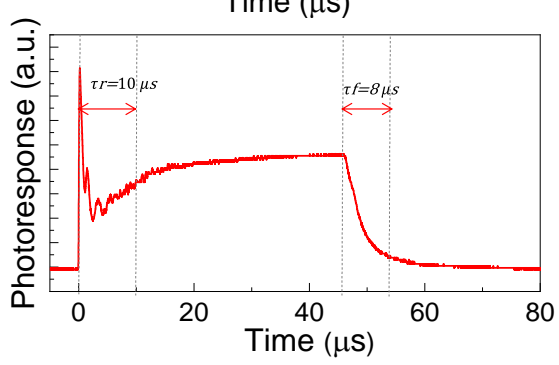

(c)

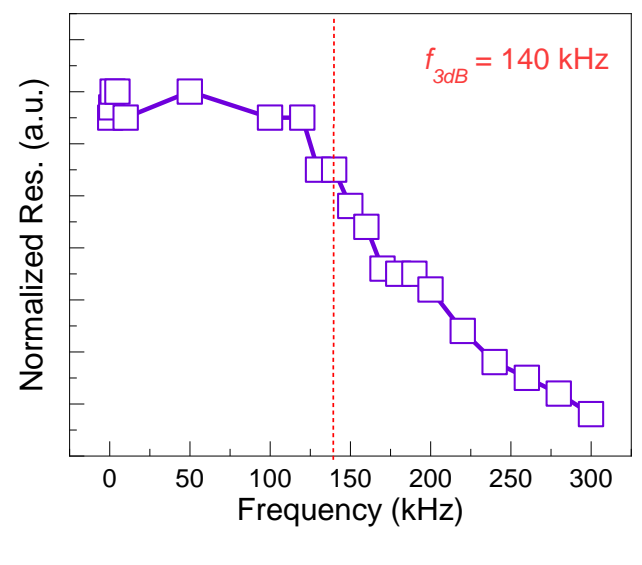

Figure S2. Response time characteristic of the device. (a) Multi-cycle photocurrent responses to periodic pulsed laser. (b) Time response of the device calculated by plotting single cycle from figure (a) is around $8 \mu \mathrm{s}$. (c) Frequency response of the device. The $3 \mathrm{~dB}$ frequency is around $140 \mathrm{kHz}$. 
(a)

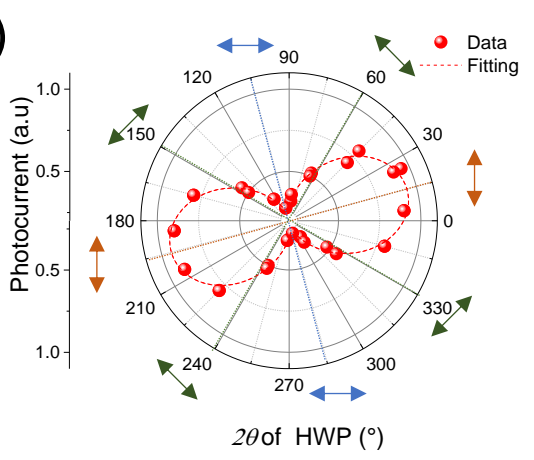

(c)
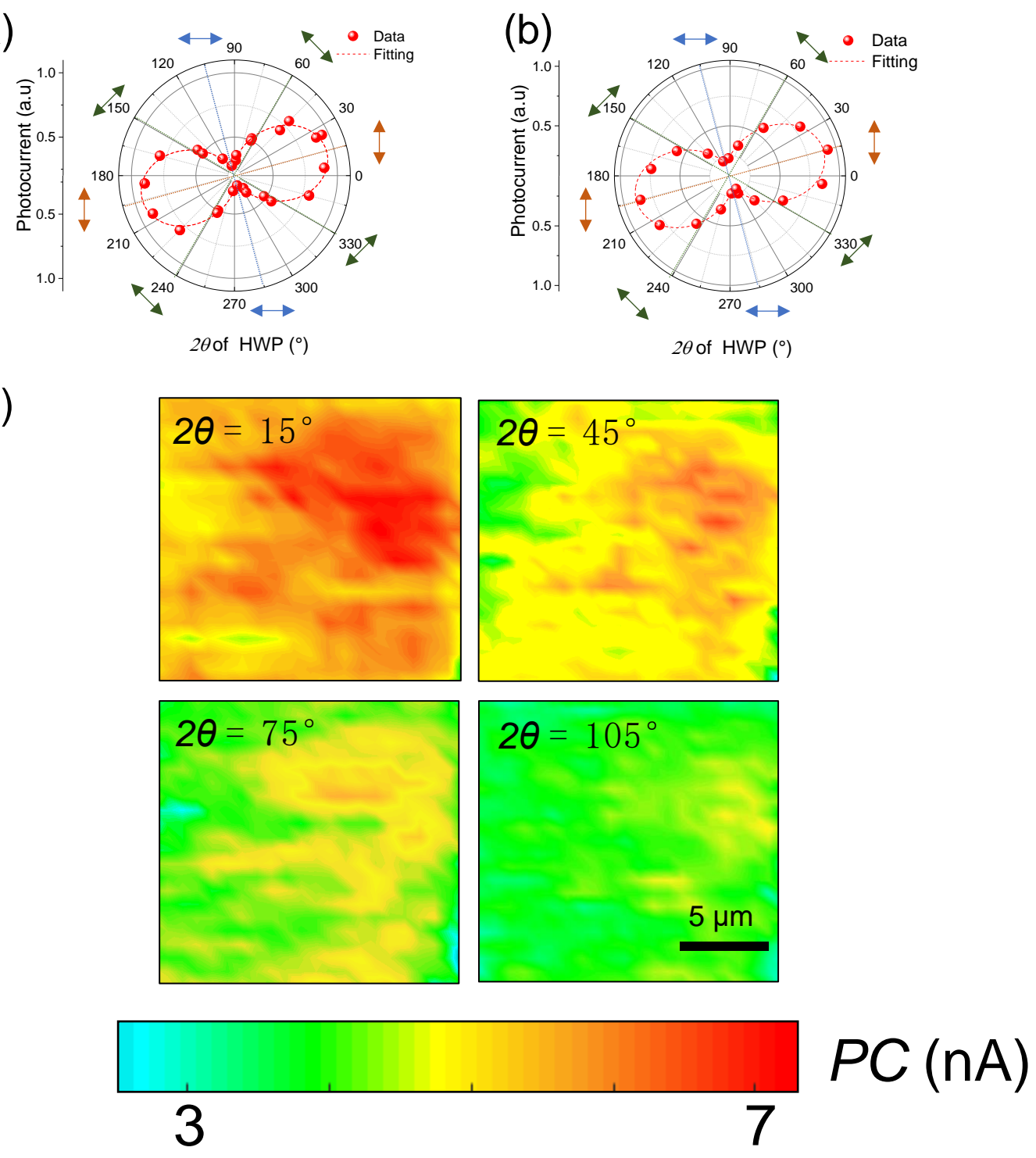

Figure S3. Linear polarization-resolved photoresponse. (a-b) Polar plots of two devices that show the photoresponse as a function of polarization angle under linearly polarized $1550 \mathrm{~nm}$ illumination. Significant two-fold mirror symmetry arises from the anisotropic morphology of the plasmonic nanostructures. The contrast ratio for the orthogonal linear polarization is 6 and 3.8 as shown in (a) and (b) respectively. (c) Photocurrent mapping of a single pixel when the direction of the linearly polarized light (equals to $2 \theta$ of HWP) is rotated for $90^{\circ}$. 


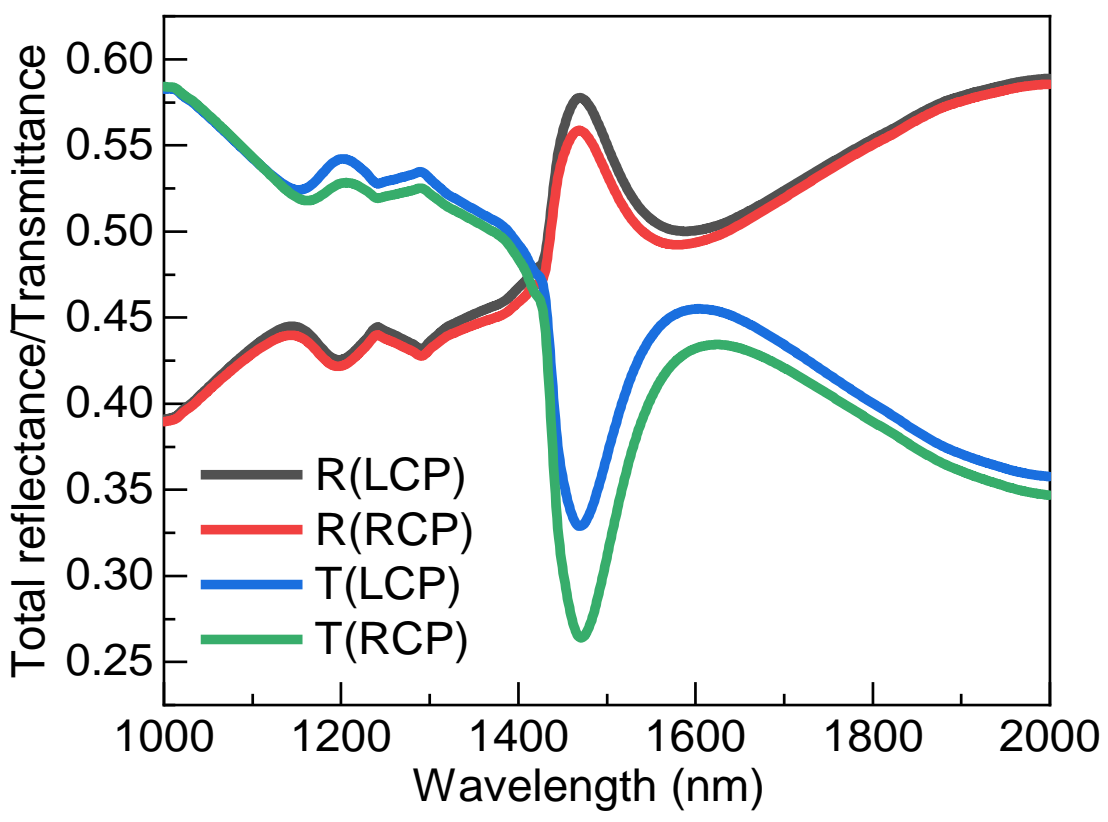

Figure S4. The total reflectance and transmittance spectra of the right-handed metasurface when it is illuminated by LCP and RCP light, respectively. Total reflectance $(\mathrm{R})$ includes both co- and cross- polarized components in the reflected waves. Total transmittance $(\mathrm{T})$ is a sum of transmitted power of all diffraction orders. 


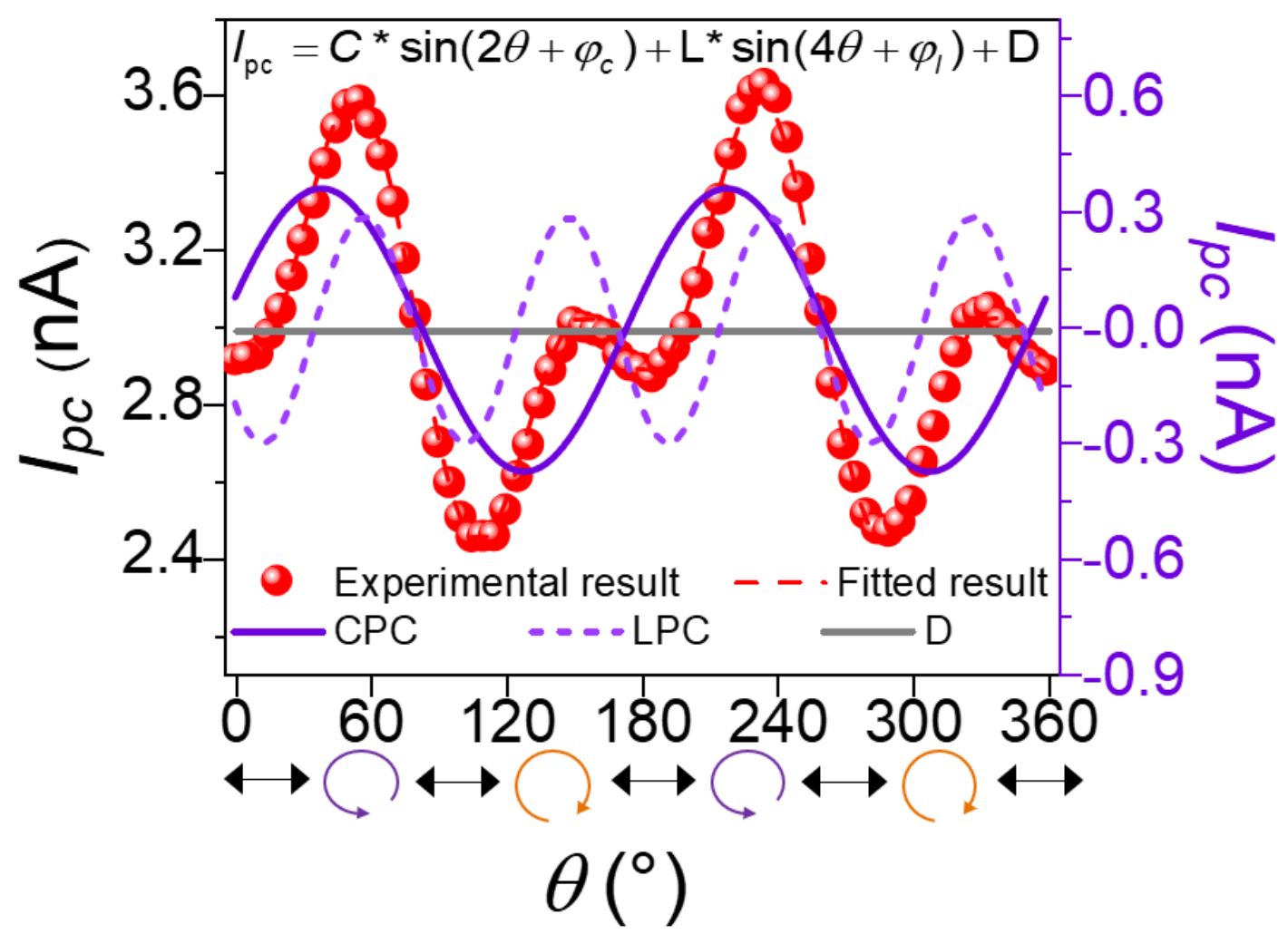

Figure S5. Retrieved linear and circular components of the photoresponse of a single pixel photodetector. The red spheres correspond to experimental data. The red dashed line is the fitting result according to the formula $I_{p c}=C \sin \left(2 \theta+\varphi_{c}\right)+L \sin \left(4 \theta+\varphi_{l}\right)+D$. The red, purple and grey solid lines represent the fitting of linear-polarized component (LPC), circular-polarized component (CPC), and constant component D, respectively. 


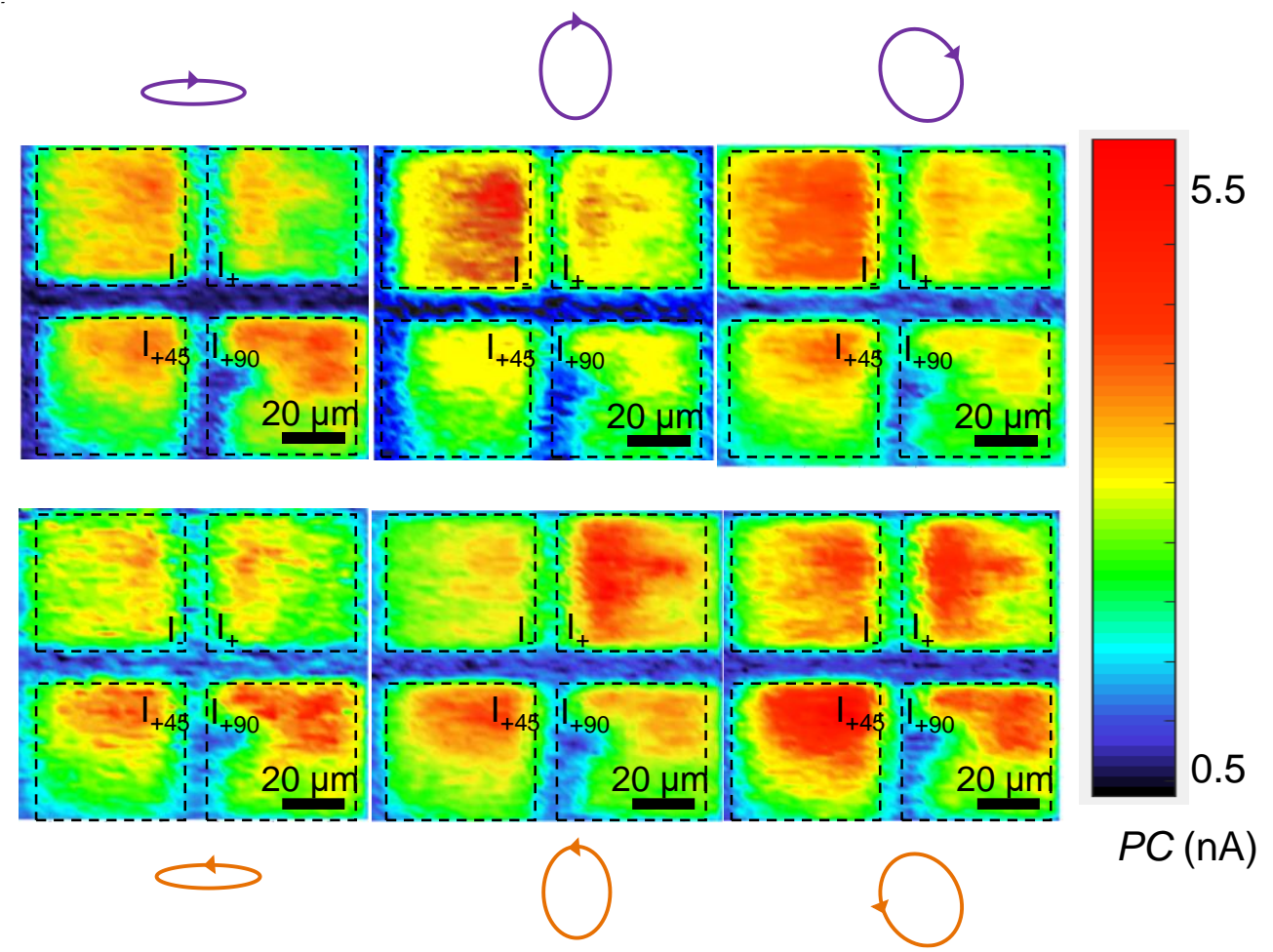

Figure S6. Scanning photocurrent microscopy results of the device at different incident polarization states. The corresponding polarization states are schematically depicted by the inset schematic on each ellipse (2.8 mW @ $1550 \mathrm{~nm},-0.6 \mathrm{~V}$, with photocurrent shown in absolute value by a color-bar). 
(a)

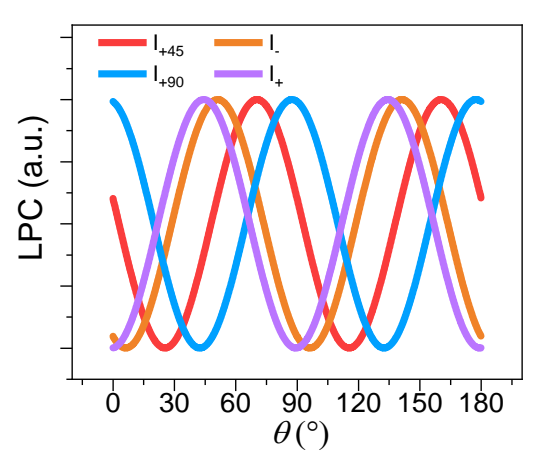

(b)

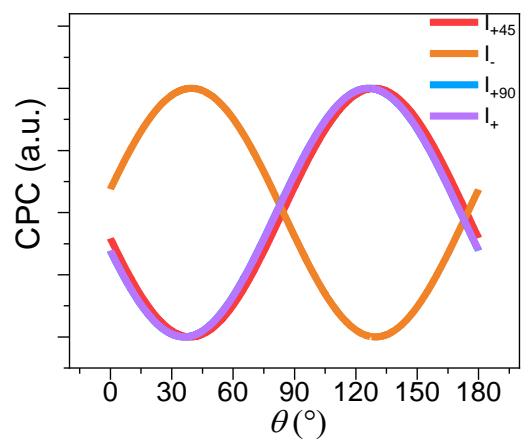

Figure S7. The obtained linearly polarized components LPC (a) and circularly polarized components CPC (b) of the photocurrent as a result of fitting in Figure $3 \mathrm{~d}$. The CPC of the $I$ - and $I_{+}\left(I_{45}, I_{90}\right)$ have a phase difference around $\pi / 2$. The LPC of the three pixels $\left(I_{+}, I_{45}, I_{90}\right)$ have a $\pi / 8(\pi / 4)$ phase difference. The small LPC difference between $I-$ and $I_{+}$is due to the higher-order harmonics component caused by the asymmetry design of the metasurfaces.

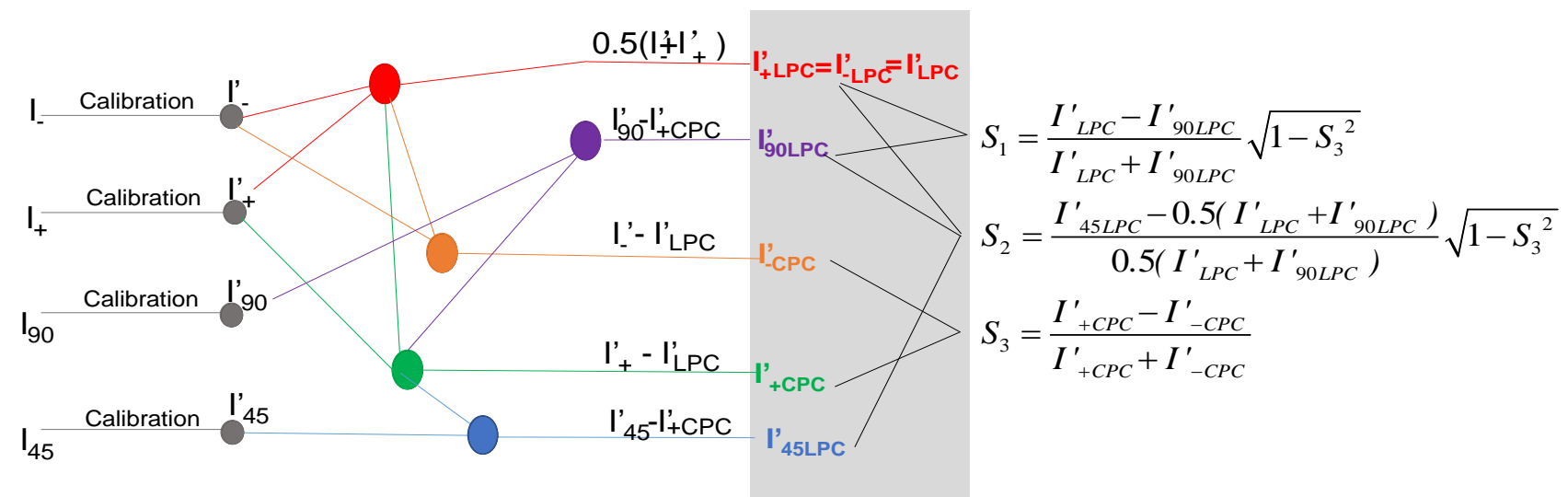

Figure S8. The illustration of the polarimetry algorithm. 
(a)

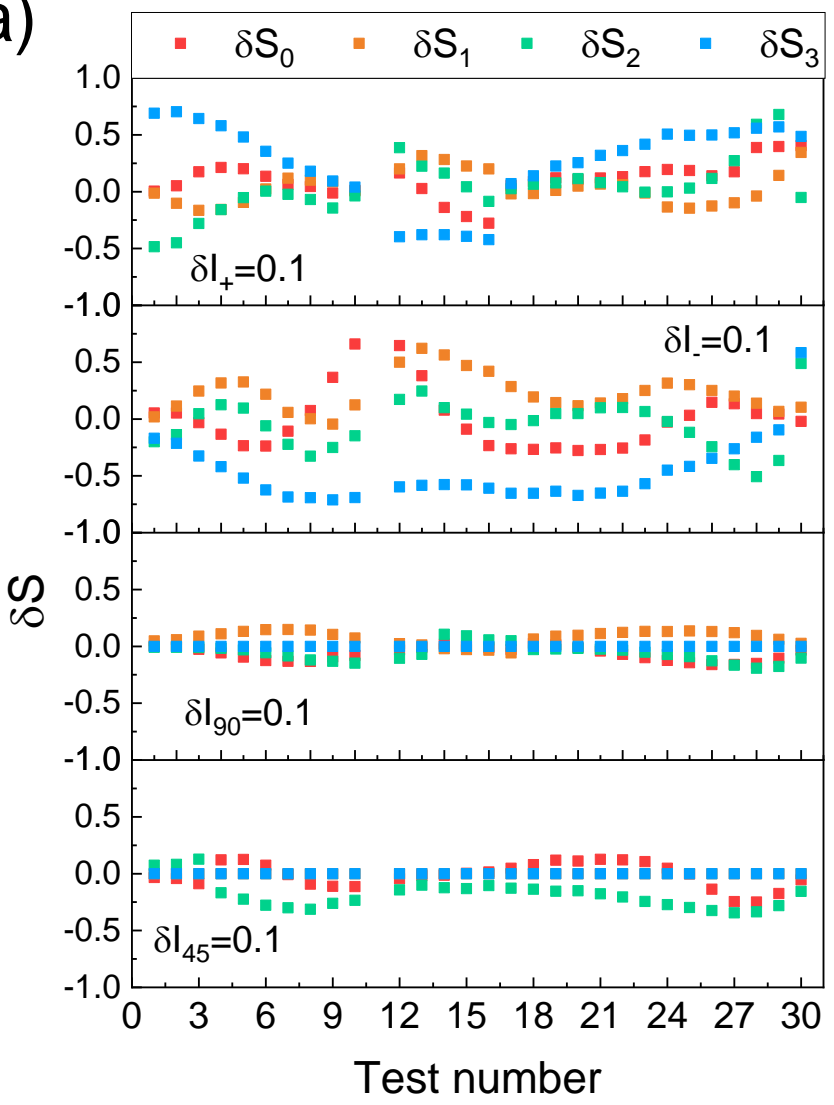

(b)

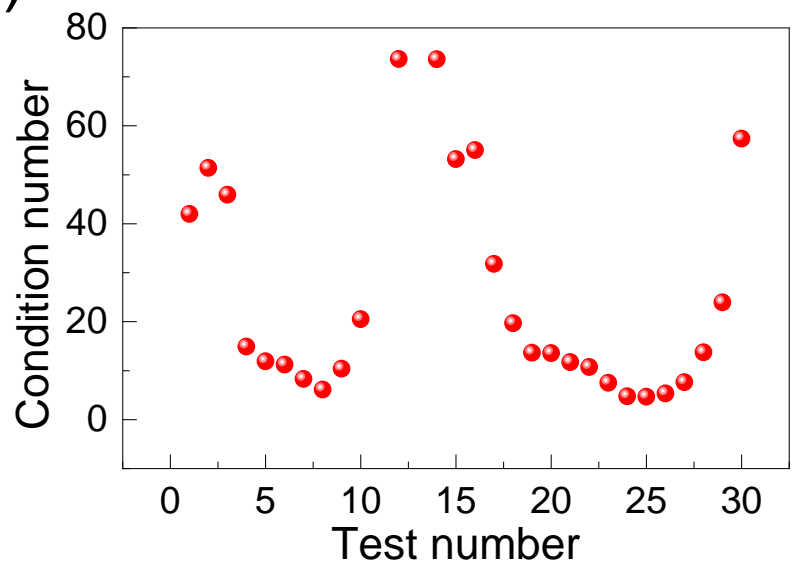

Figure S9. (a) The variation in outputs at different inputs perturbation. (b) The local condition number at different inputs. 


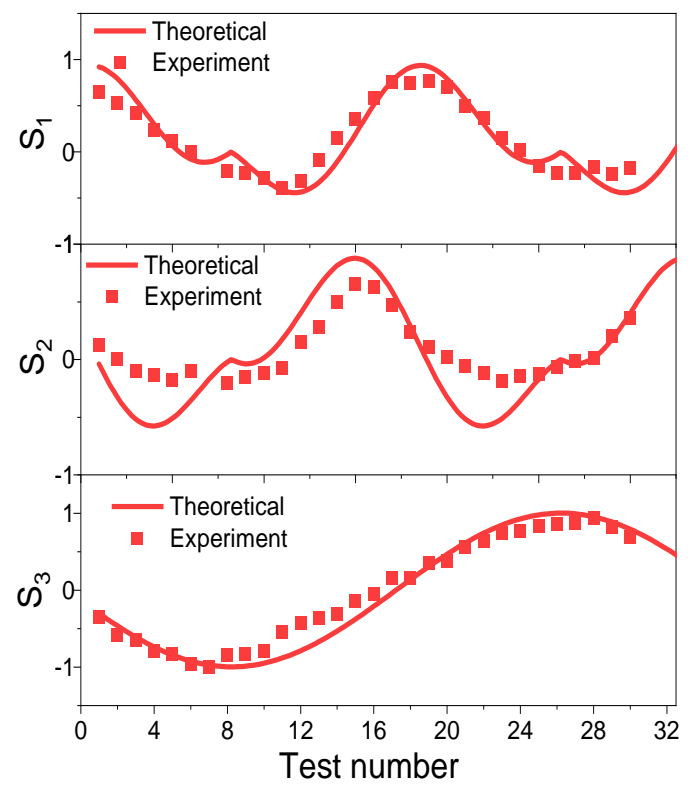

Figure S10. The retrieved Stokes parameters with elliptically polarized inputs. 
(a)

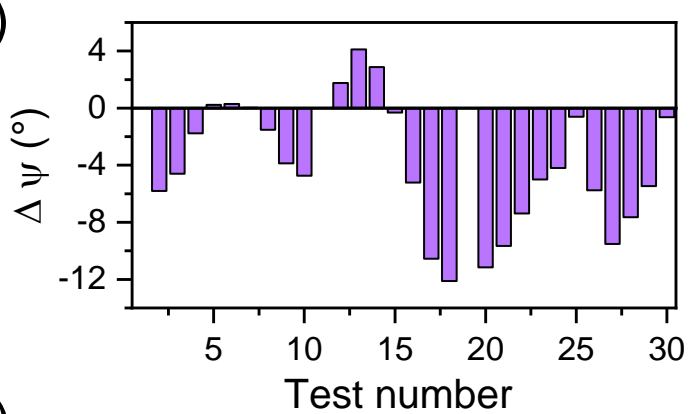

(b)

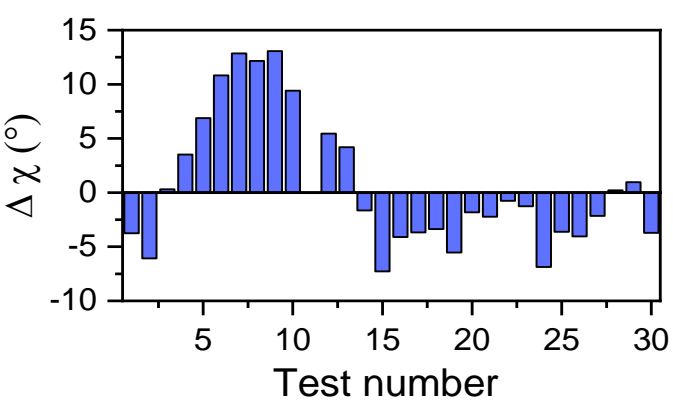

Figure S11. The analysis of deviation in (a) azimuthal angle $\psi$ and (b) ellipticity angle $\chi$ from Fig.4b.

(a)

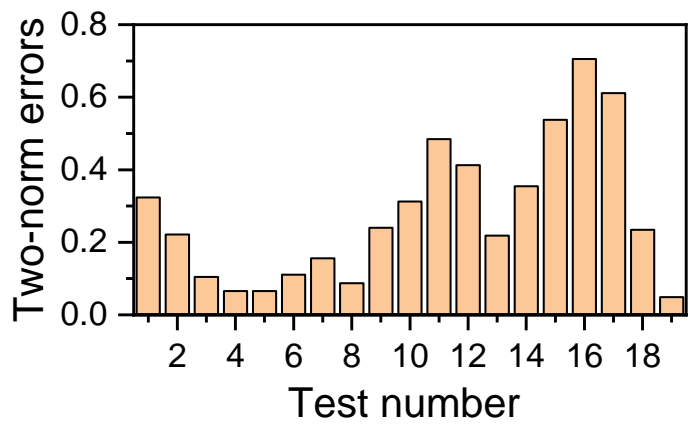

(b)

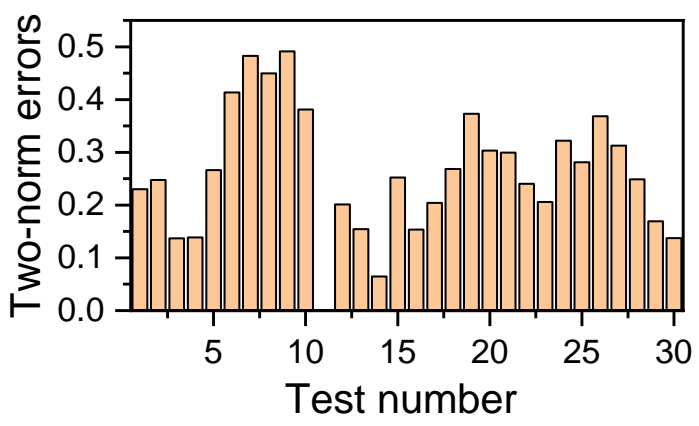

Figure S12. The calculated two-norm errors in Figure 4a \&b respectively. 

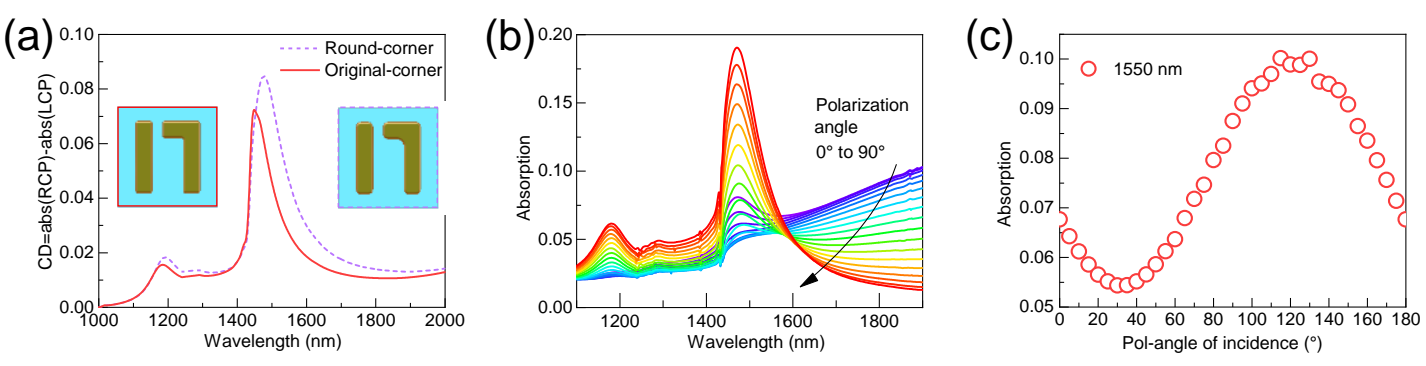

Figure S13. The polarimetry deviation from the plasmonic-antenna. (a) The CD spectra of the metasurfaces with round-corner (purple dashed line) and original-corner (red solid line). (b) The total absorption of the metasurfaces at different linearly-polarization angle. (c) The absorption at $1550 \mathrm{~nm}$ as a function of the polarization angle of the incident wave at normal incidence. 


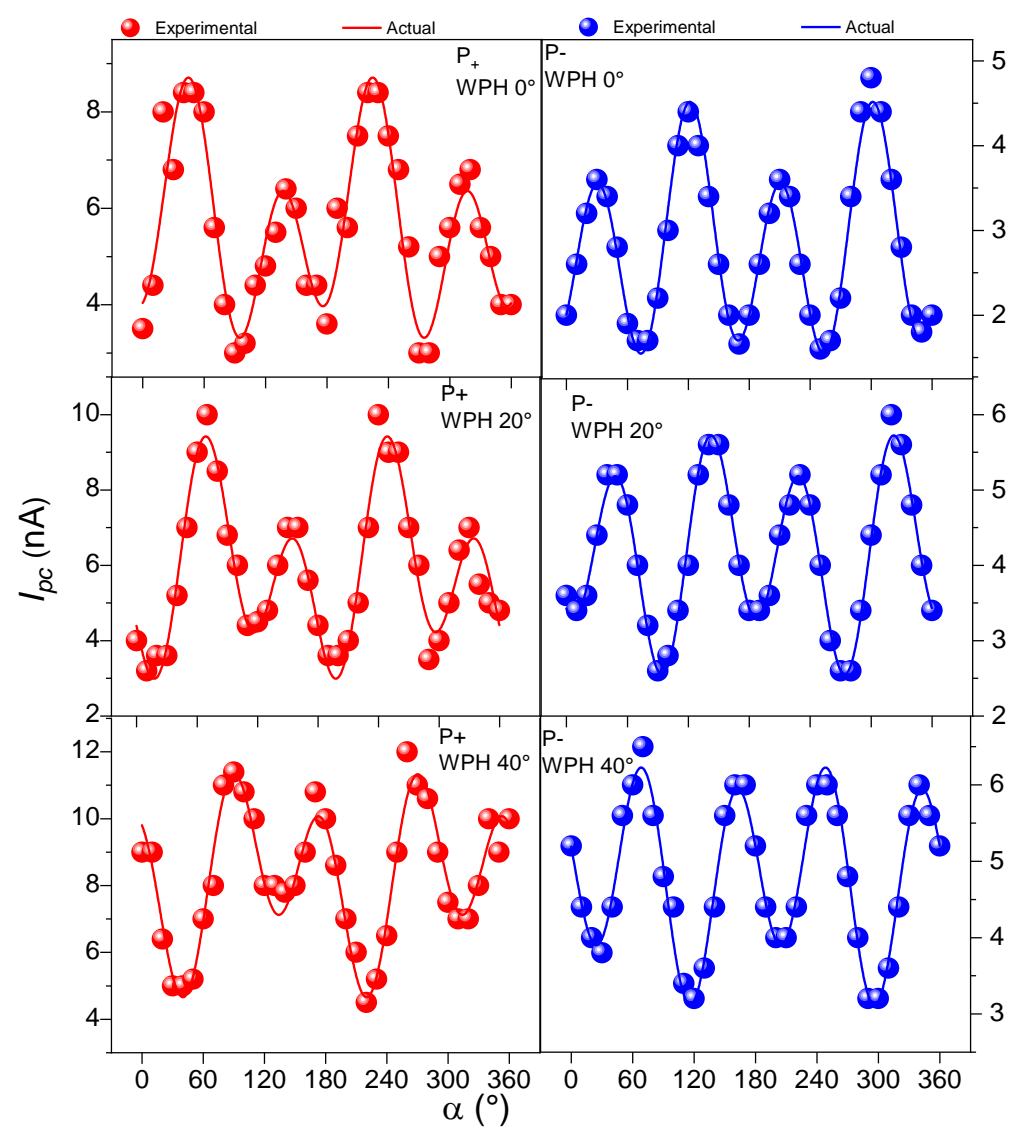

Figure S14. Polarization-resolved characteristics for $2 \mu \mathrm{m}^{2}$ scale pixel.

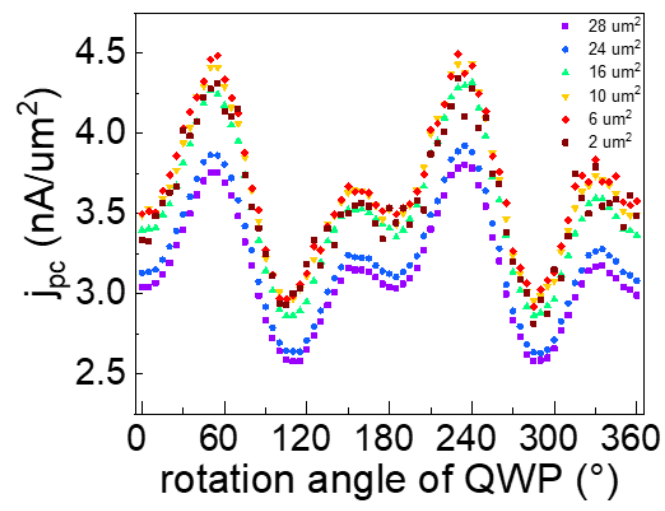

Figure S15. The photocurrent density as a function of incident light polarization states (controlled by rotation angle of QWP) at different effective spot size illumination. 
(a)

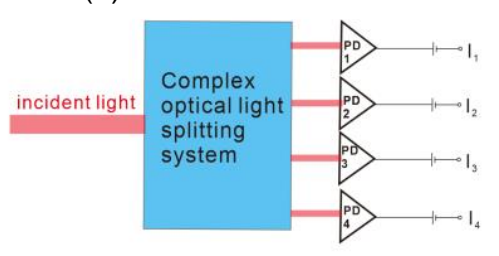

(b)

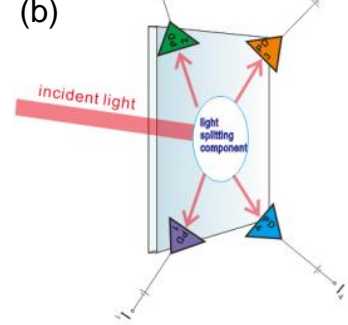

(c)

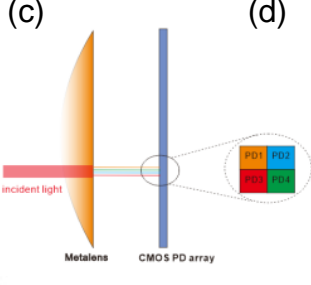

(d)

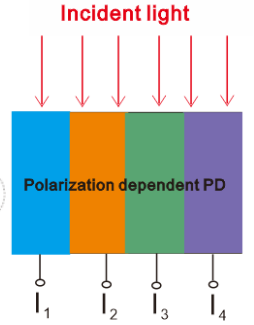

Figure S16. The schematics of different polarimetry strategies. (a) Traditional polarimeter system based on complex optical path design. (b) Polarimeter based on onchip light-splitting structure (waveguide or metasurface intervene structure). (c) Polarimeter based on plasmonic metalens. (d) Polarimeter based on polarization sensitive photodetector. 


\section{Supplementary Text.}

\section{The influence of contrast ratio on the polarimetry.}

In principle, the computational extraction of Stokes parameters in our device does not rely on the absolute value of $\mathrm{CD}$ (the contrast ratio between different polarization states), which is a major advantage of our device design. The key point is: The polarization-dependent photocurrent in our device has been modeled, and prior knowledge about the non-polarized photocurrent can be obtained and then removed from the total photocurrent through a simple calibration process. Which is discussed as below.

Generally, exciting with a quarter wave plate (QWP) modulated linearly polarized laser, the total photocurrent of a detector can be decomposed into three components: the linear-polarization dependent component $\left(I_{l p c}\right)$, the circular-polarization dependent component $\left(I_{c p c}\right)$, and polarization independent component D (as shown in Eq. S1, the derivation of Eq. S1 is in Supplementary Text 2).

$$
\begin{aligned}
& I_{+}=I_{+c p c}+I_{+l p c}+D=C \sin \left(2 \theta+\varphi_{c+}\right)+L \sin \left(4 \theta+\varphi_{l+}\right)+D \\
& I_{-}=I_{-c p c}+I_{-l p c}+D=C \sin \left(2 \theta+\varphi_{c-}\right)+L \sin \left(4 \theta+\varphi_{l-}\right)+D \\
& I_{90}=I_{90 c p c}+I_{90 l p c}+D=C \sin \left(2 \theta+\varphi_{c 90}\right)+L \sin \left(4 \theta+\varphi_{l 90}\right)+D \\
& I_{45}=I_{45 c p c}+I_{45 l p c}+D=C \sin \left(2 \theta+\varphi_{c 45}\right)+L \sin \left(4 \theta+\varphi_{l 45}\right)+D
\end{aligned}
$$

Therefore, a large $\mathrm{D}$ component decreases the photocurrent contrast ratio between the polarized and non-polarized states (or equally the CD). For instance, Figure S5 shows the total photocurrent and its three components of a typical pixel. The polarization independent component $\mathrm{D}$ occupies a large portion of the total photocurrent, resulting in a relatively small CD.

It can be also seen from the Figure S5 that the model can explain the experimental 
results. Through a calibration process in which we put a QWP in front of a laser source and then measured the photo-responsivity as a function of the rotation angle of the wave plate (such as in Figure S5), a prior knowledge of the polarization independent component $\mathrm{D}$ can be obtained by fitting the results to Eq. S1. By using the calibration method, the CD does not affect the extraction of Stokes parameters. In addition, in our device, the four pixels have the same metasurfaces geometry. Hence, the proportion of D is nearly the same among the four pixels. All the pixels can share a same calibration, which largely simplify the calibration process. Hence, we conclude that the limited contrast ratio between different circular polarization states will not degrade the polarimetry performance.

Definitely, from the experimental point of view, a higher contrast ratio enhances the polarimetry accuracy in experiments. The reason is as follows: Besides the three components in Eq. S1, an additional remainder term R exists practically (Eq. S2). The $\mathrm{R}$ arises from non-ideal effects such as the high-order harmonic term (induced by nonlinear polarization states resulted from asymmetric geometry of the metasurfaces) and detector noise. The remaining terms are generally different among four pixels. Hence it cannot be eliminated during our computational reconstruction. As a result, the obtained LPC and CPC have an additional error term induced by the R. In this condition, the enhancement of contrast ratio (equivalent to the decrease of $\mathrm{R}$ ) improves the polarimetry accuracy.

$$
\begin{aligned}
& I_{+}=C \sin \left(2 \theta+\varphi_{c+}\right)+L \sin \left(4 \theta+\varphi_{l+}\right)+D+R_{+} \\
& I_{-}=C \sin \left(2 \theta+\varphi_{c-}\right)+L \sin \left(4 \theta+\varphi_{l_{-}}\right)+D+R_{-} \\
& I_{90}=C \sin \left(2 \theta+\varphi_{c 90}\right)+L \sin \left(4 \theta+\varphi_{l 90}\right)+D+R_{90} \\
& I_{45}=C \sin \left(2 \theta+\varphi_{c 45}\right)+L \sin \left(4 \theta+\varphi_{l 45}\right)+D+R_{45}
\end{aligned}
$$

To improve the contrast ratio, optimization can be considered from two aspects: 
a) Optimizing the design of metasurfaces. Though the metasurfaces in this manuscript successfully demonstrate polarimetry but can be further optimized for improvement in the performance. For example, to reduce the non-ideal factors, metasurfaces with higher symmetry may be more suitable.

b) Enhancing the sensitivity (signal-to-noise ratio) of photodetectors. There are several strategies to achieve this goal. Firstly, optimizing the transport property of the detector. The common strategy is to improve the interfaces. There are two key interfaces in our device, the graphene/metasurface interface and the silicon/metasurface interface. The non-ideal interface not only impedes the light absorption, but also hinders the carrier transport process. Secondly, increasing the Q-factor of the plasmonic antenna can also enhance sensitivity. Higher Q-factor means smaller energy-loss. The Q-factor can be increased by optimizing the uniformity and geometrical accuracy of the metasurfaces during the fabrication process. The distorted shape and nonuniform distribution of metasurfaces broaden the actual plasmonic resonant peak and reduce Q-factor.

\section{Algorithm for retrieving Stokes parameters.}

As we designed, the photocurrent of each pixel is both circularly (due to the chirality) and linearly (due to anisotropy) polarization dependent. The general expression of the photocurrents can be expressed as

$$
\begin{aligned}
& I_{+}=I_{+c p c}+I_{+l p c}+D=C \sin \left(\alpha+\varphi_{c+}\right)+L \sin \left(\beta+\varphi_{l+}\right)+D \\
& I_{-}=I_{-c p c}+I_{-l p c}+D=C \sin \left(\alpha+\varphi_{c-}\right)+L \sin \left(\beta+\varphi_{l-}\right)+D \\
& I_{90}=I_{90 c p c}+I_{90 l p c}+D=C \sin \left(\alpha+\varphi_{c 90}\right)+L \sin \left(\beta+\varphi_{l 90}\right)+D \\
& I_{45}=I_{45 c p c}+I_{45 l p c}+D=C \sin \left(\alpha+\varphi_{c 45}\right)+L \sin \left(\beta+\varphi_{l 45}\right)+D
\end{aligned}
$$


Physically, the angle variables $\alpha$ and $\beta$ together with the phase factor $\varphi$, represent the polarization angle of the incident light in each metasurface pixel. For linearly polarized component, the polarization angle $\beta+\varphi$ is the angle between the long axis of the metasurface and the polarization direction of the light. For circularly polarized component, $C \sin (\alpha+\varphi)$ represents the eccentricity of the elliptically polarized incident light.

Experimentally, to verify this polarization dependence, we adapted a wellestablished method from the studies of circularly polarized photo-galvanic effect, ${ }^{1-3}$ in which the linearly polarized laser source is modulated by a QWP. By rotating the QWP, the incident linearly polarized light is converted to different elliptical polarization states. In addition, the eccentricity and the long axis of the elliptically polarized light are correlated by the rotating angle $\theta$, i.e. $\alpha=2 \theta$ and $\beta=4 \theta$. Therefore, we can simplify the Eq. S3 as

$$
\begin{aligned}
& I_{+}=C \sin \left(2 \theta+\varphi_{c+}\right)+L \sin \left(4 \theta+\varphi_{l+}\right)+D \\
& I_{-}=C \sin \left(2 \theta+\varphi_{c-}\right)+L \sin \left(4 \theta+\varphi_{l-}\right)+D \\
& I_{90}=C \sin \left(2 \theta+\varphi_{c 90}\right)+L \sin \left(4 \theta+\varphi_{l 90}\right)+D \\
& I_{45}=C \sin \left(2 \theta+\varphi_{c 45}\right)+L \sin \left(4 \theta+\varphi_{l 45}\right)+D
\end{aligned}
$$

We would like to point out that experimental results agree with our photocurrent expression. As demonstrated in Figure S5, when the QWP rotates for 360 degree, the photocurrent (of a typical pixel) changes periodically. The overall photocurrent accurately fits to the expression Eq. S4 (Figure S7 also exemplifies the fitting to all the 4 pixels of a device). The fitting shows that $I_{p c}$ is comprised of three terms as the QWP rotates. The term $C \sin \left(2 \theta+\varphi_{c}\right)$ (the purple solid line in Figure S5) consists with the ellipticity of the incident light experimentally and has a $\pi / 2$ phase difference between pixel $P_{+}$and $P_{\text {. (that is }} \varphi_{c^{+}}-\varphi_{c^{-}}=\pi / 2$ ). Therefore, we conclude that it represents the 
helicity-resolved component of the incident light. The second periodic term $L \sin \left(4 \theta+\varphi_{l}\right)$ (the purple dashed line in Figure S5) consists of the change of the direction of the long axis of the incident elliptical-polarized light. Therefore, it represents the linear polarization-resolved component in total photocurrent. The last term $D$ (the grey solid line in Figure S5) is a polarization-independent constant background response.

As aforementioned, the constant parameters (C, L, and D) can be obtained by calibrating one of these pixels. Because of the similarity in fabrication and design, the calibrated results can be shared with other pixels. Therefore, we can normalize the above photocurrents as

$$
\begin{aligned}
& I_{+}{ }^{\prime}=I_{+c p c}^{\prime}+I_{l p c}^{\prime}=C\left[\sin \left(\alpha+\varphi_{c+}\right)+1\right]+L\left[\sin \left(\beta+\varphi_{l+}\right)+1\right] \\
& I_{-}{ }^{\prime}=I^{\prime}{ }_{-p p c}+I_{l p c}^{\prime}=C\left[\sin \left(\alpha+\varphi_{c-}\right)+1\right]+L\left[\sin \left(\beta+\varphi_{l-}\right)+1\right] \\
& I_{90}{ }^{\prime}=I_{90 c p c}^{\prime}+I_{90 l p c}^{\prime}=C\left[\sin \left(\alpha+\varphi_{c 90}\right)+1\right]+L\left[\sin \left(\beta+\varphi_{l 90}\right)+1\right] \\
& I_{45}{ }^{\prime}=I_{45 c p c}^{\prime}+I_{45 l p c}^{\prime}=C\left[\sin \left(\alpha+\varphi_{c 45}\right)+1\right]+L\left[\sin \left(\beta+\varphi_{l 45}\right)+1\right]
\end{aligned}
$$

The Eq. S5 normalizes the photocurrents of each pixel into two parts: the circular polarization dependent photocurrent $\left(I_{+c p c}^{I^{\prime}}\right.$ and $\left.I_{-c p c}^{\prime}\right)$ and the linear polarization dependent photocurrent $\left(I^{\prime}{ }_{45 l p c}, I_{90 l p c}^{\prime}, I_{l p c}^{\prime}\right)$. After calibration and normalization, we extracted the Stoke parameters from these polarization-dependent photocurrents as illustrated in Figure S8.

Finally, we summarize the explicit solutions of the polarimetry algorithm in Eq. S6-9 as below:

$$
\begin{aligned}
& S_{0}{ }^{2}=S_{1}{ }^{2}+S_{2}{ }^{2}+S_{3}{ }^{2} \\
& S_{1}=\frac{I_{L P C}^{\prime}-I_{90 L P C}^{\prime}}{I_{L P C}^{\prime}+I_{90 L P C}^{\prime}} \sqrt{1-S_{3}{ }^{2}} \\
& S_{2}=\frac{I_{45 L P C}^{\prime}-0.5\left(I_{L P C}^{\prime}+I_{90 L P C}^{\prime}\right)}{0.5\left(I^{\prime}{ }_{L P C}+I_{90 L P C}^{\prime}\right)} \sqrt{1-S_{3}{ }^{2}}
\end{aligned}
$$




$$
S_{3}=\frac{I_{+C P C}^{\prime}-I_{-C P C}^{\prime}}{I^{\prime}{ }_{+C P C}+I^{\prime}{ }_{-C P C}}
$$

Therefore, we cannot simply obtain a linear polarimetric matrix $M$ and define a global condition number by $\left[\begin{array}{c}S_{0} \\ S_{1} \\ S_{2} \\ S_{3}\end{array}\right]=[M]\left[\begin{array}{c}I_{+} \\ I_{-} \\ I_{45} \\ I_{90}\end{array}\right]$. Instead of that, we write the non-linear relationship between the inputs and the outputs (according to Eq. S6-9) as $\left[\begin{array}{l}S_{0} \\ S_{1} \\ S_{2} \\ S_{3}\end{array}\right]=f\left(\left[\begin{array}{c}I_{+} \\ I_{-} \\ I_{45} \\ I_{90}\end{array}\right]\right)$. To evaluate the sensitivity of the outputs $\left(S_{0}, S_{1}, S_{2}, S_{3}\right)$ to small perturbations in the input data $\left(I_{+}, I_{-}, I_{45}, I_{90}\right)$ for this non-linear problem, we define a "local" matrix M' (which means the matrix and condition number is varied for different Stokes parameters) by

$$
\left[\begin{array}{c}
S_{0} \\
S_{1} \\
S_{2} \\
S_{3}
\end{array}\right]=f\left(\left[\begin{array}{c}
I_{+} \\
I_{-} \\
I_{45} \\
I_{90}
\end{array}\right]\right) \rightarrow M^{\prime}=\left[\begin{array}{llll}
\frac{\partial S_{0}}{\partial I_{+}} & \frac{\partial S_{0}}{\partial I_{-}} & \frac{\partial S_{0}}{\partial I_{45}} & \frac{\partial S_{0}}{\partial I_{90}} \\
\frac{\partial S_{1}}{\partial I_{+}} & \frac{\partial S_{1}}{\partial I_{-}} & \frac{\partial S_{1}}{\partial I_{45}} & \frac{\partial S_{1}}{\partial I_{90}} \\
\frac{\partial S_{2}}{\partial I_{+}} & \frac{\partial S_{2}}{\partial I_{-}} & \frac{\partial S_{2}}{\partial I_{45}} & \frac{\partial S_{2}}{\partial I_{90}} \\
\frac{\partial S_{3}}{\partial I_{+}} & \frac{\partial S_{3}}{\partial I_{-}} & \frac{\partial S_{3}}{\partial I_{45}} & \frac{\partial S_{3}}{\partial I_{90}}
\end{array}\right]
$$

For each input, we can obtain a matrix M', which represents the variation in the outputs $\left(S_{0}, S_{1}, S_{2}, S_{3}\right)$ to the change in the inputs $\left(I_{+}, I_{-}, I_{45}, I_{90}\right)$. It is not difficult to find this continuation is very similar to the linear case. We can evaluate the robustness and stability of our algorithm by calculating the condition number of $\mathrm{M}^{\prime}$ at each input. 
In this way, we numerically calculate the matrix and condition number for the Stokes parameters measured in Figure 4b. The matrix elements of M' (or equally, the partial derivate in Eq. S10) for each test were shown in Figure S9a. The local condition numbers at each test were also shown in Figure S9b. Please note that although the conditional numbers (especially for some discrete points) dramatically increase, they are close to the previous reports. ${ }^{4,5}$

\section{The analysis on the polarimetry accuracy.}

The polarimetry accuracy can be evaluated by two strategies. The first strategy is evaluating the deviation by the degree of polarization, azimuthal angle $\psi$, and ellipticity angle $\chi$. We assessed the accuracy of a set of measured results (Fig. 4b) and demonstrated it in Figure S11 (The degree of polarization is not discussed because the inputs are fully polarized light).

We also calculated the two-norm errors of the Stokes parameters $\left(S_{1}, S_{2}, S_{3}\right)$, which is another strategy to evaluate the deviation directly from the Poincare sphere. We calculated the two-norm error by

$$
E=\sum_{i=1,2,3} \sqrt{\left(S_{i, E}-S_{1, T}\right)^{2}}
$$

where $S_{i, E}$ and $S_{i, T}$ stand for the experimental and theoretical values of the $i$ th Stokes parameters, respectively. The results of the two devices (Figure $4 a \& b$ ) are shown in Figure S12. 


\section{The polarimetry deviation from the plasmonic-antenna design.}

We first consider the effect of imperfect fabrication by modeling nano-antenna with structural distortion. While the CD spectral slightly changes, the field distribution does not change. As a result, the CPGE phase should not change (Figure S13a). We then examined the LPGE effect of the anisotropic nano antenna. To test the anisotropy of the structure, we illuminated the structure with linearly polarized waves and varied the polarization angle in the simulation. Figure S13b shows the calculated total absorption of the nano-antenna. The absorption spectra are highly anisotropic. We summarized the absorption at $1550 \mathrm{~nm}$ as a function of the polarization angle of the incident wave at normal incidence in Figure S13c. The optical absorption function has clearly deviated from a normal sinusoidal function. Mathematically, this deviation can be expressed by a sum of higher-order harmonics. Accordingly, these undesired higherorder harmonics result in a computational error.

These higher-order harmonics are easy to be understood if we consider the structure of the nano-antenna. The lack of centrosymmetry distorts the optical response function from a perfect sinusoidal function. To improve the accuracy, we should choose antenna shape with better symmetry.

\section{Polarization-resolved characteristics for micrometer-scale pixel.}

Although our demonstrated photodector is around 10 20 $\mu \mathrm{m}$ in size (as in Figure 1c), it keeps the polarimetry function without noticeable performance degradation even when the pixel size scales down to few micrometers. As demonstrated in Figure S14, when the infrared laser is fixed on a $2 \mu \mathrm{m}$-size pixel region, the experimental results 
(the solid spheres) are consistent with the real polarization states (the solid lines). We also studied the pixel-size dependence of device performance (Figure S15), and devices depicted good consistency in polarimetry when the pixel-size varies from $28 \mu \mathrm{m}^{2}$ to 2 $\mu \mathrm{m}^{2}$. Theoretically, the spot size of the laser source has a negligible influence on the polarimetry performance if the laser power density, nano-antenna size, and graphene quality are uniform. Experimentally, some non-ideal factors such as the non-uniformity among plasmonic units will induce a size-dependent noise term. As a result, the photodetector performance, especially the signal-to-noise ratio may change with laser size, as demonstrated in Figure S15. However, the key performances, including the polarimetry functionality, will not change noticeably.

\section{Comparison of our four-pixel polarimeter with recent works.}

Many miniature polarimeters have been proposed recently (Figure S16a), which rely on sophisticate light path design and expensive optical components. Typical strategies include on-chip light splitting structure (Figure S16b), metalens with light splitting and polarization-dependent focusing components (Figure S16c), and polarization-sensitive photodetectors (Figure S16d). For the polarimeter based on the metalens, it is very difficult to realize on-chip integration because the metalens should be separated from electrical-readout array spatially in light path to allow light focusing, which largely limits its potential application in future on-chip photonic and optoelectronic integration system. For the on-chip light splitting strategy, although it is theoretically feasible to integrate the light splitting components and electrical sensors on chip, the integration level and the signal intensity will be compromised due to the 
complexity and large size of the splitting components. Besides, the applications may be also be limited because of serious interference between neighboring pixels.

In comparison, the polarization-sensitive photodetector has two obvious advantages: Firstly, our design is ultracompact. The polarization-sensitive component (metasurfaces) and compact photodetectors are naturally integrated together, which largely improves the device footprint and integration level. Second, each pixel combines the functionalities of polarization sensing and electrical readout, which greatly simplifies the optical design and reduces signal loss during signal conversion and transport. As summarized in Table S1, our work mostly outperforms previous works in terms of footprint. 


\section{Supplementary Table}

\begin{tabular}{|c|c|c|c|c|c|c|c|}
\hline $\begin{array}{l}\text { Device } \\
\text { Structures }\end{array}$ & $\begin{array}{l}\text { CPC } \\
\text { detecti } \\
\text { on }\end{array}$ & $\begin{array}{l}\text { LPC } \\
\text { detecti } \\
\text { on }\end{array}$ & $\begin{array}{l}\text { Mechanism } \\
\text { for } \\
\text { polarization } \\
\text { detection }\end{array}$ & $\begin{array}{l}\text { Demonstrat } \\
\text { ed } \\
\text { detection } \\
\text { wavelength }\end{array}$ & $\begin{array}{l}\text { Wheth } \\
\text { er on- } \\
\text { chip }\end{array}$ & $\begin{array}{l}\text { minim } \\
\text { al } \\
\text { pixel } \\
\text { size }\end{array}$ & $\begin{array}{l}\text { Full-Stokes } \\
\text { Polarimetry }\end{array}$ \\
\hline $\begin{array}{l}\text { Dielectric } \\
\text { metasurfaces } \\
\text { lens }^{6}\end{array}$ & Q & Q & $\begin{array}{l}\text { splitting and } \\
\text { focusing } \\
\text { light }\end{array}$ & $850 \mathrm{~nm}$ & \multicolumn{2}{|c|}{ Not On-chip } & प \\
\hline $\begin{array}{l}\text { Dielectric } \\
\text { metasurfaces } \\
\text { lens }{ }^{7}\end{array}$ & Q & ૫ & (Fig. S14c) & $\sim 532 \mathrm{~nm}$ & \multicolumn{2}{|c|}{ Not On-chip } & प \\
\hline $\begin{array}{l}\text { Photonic } \\
\text { crystal-like } \\
\text { light splitter }\end{array}$ & Q & ૫ & \multirow{3}{*}{$\begin{array}{c}\text { On-chip } \\
\text { light } \\
\text { splitting } \\
\text { (Fig. S14b) }\end{array}$} & $\sim 1550 \mathrm{~nm}$ & YES & $\sim 700 \mu \mathrm{m}$ & Q \\
\hline $\begin{array}{l}\text { Plasmonic X- } \\
\text { shaped } \\
\text { aperture } \\
\text { arrays }{ }^{9}\end{array}$ & Q & Q & & $\sim 800 \mathrm{~nm}$ & $\begin{array}{l}\text { Not } \\
\text { demonst } \\
\text { rated }\end{array}$ & $\sim 50 \mu \mathrm{m}$ & Q \\
\hline $\begin{array}{l}\text { Cirality- } \\
\text { selectived } \\
\text { plasmonic- } \\
\text { waveguide }^{10}\end{array}$ & Q & $\mathbf{X}$ & & $1550 \mathrm{~nm}$ & YES & $10 \mu \mathrm{m}$ & $\mathbf{X}$ \\
\hline $\begin{array}{l}\text { Silicon-based } \\
\text { plasmonic } \\
\text { polarimeter }^{11}\end{array}$ & Q & ૫ & \multirow{2}{*}{$\begin{array}{c}\text { SPRs } \\
\text { enhanced } \\
\text { photocurrent } \\
\text { (Fig.S14d) }\end{array}$} & $700-1000 \mathrm{~nm}$ & YES & $\begin{array}{c}\sim 5 \\
\mu \mathrm{m} * 6\end{array}$ & Q \\
\hline $\begin{array}{l}\mathrm{Ag} \\
\text { metasurface } \\
/ \text { Ti/Si } \\
\text { Junction }^{12}\end{array}$ & Q & $\mathbf{X}$ & & $\sim 1300 \mathrm{~nm}$ & YES & $\begin{array}{l}\mathrm{N} / \\
\mathrm{A}\end{array}$ & $\mathbf{X}$ \\
\hline $\begin{array}{l}\text { Black } \\
\text { phosphorus - } \\
\text { based } \\
\text { heterostructur } \\
\mathrm{e}^{13}\end{array}$ & $\mathbf{x}$ & Q & \multirow{2}{*}{$\begin{array}{l}\text { Anisotropy } \\
\text { absorption of } \\
\text { material } \\
\text { (Fig.S14d) }\end{array}$} & $\begin{array}{c}400 \sim 3750 \\
\mathrm{~nm}\end{array}$ & YES & $\begin{array}{l}\mathrm{N} / \\
\mathrm{A}\end{array}$ & $\mathbf{X}$ \\
\hline $\begin{array}{l}\text { Graphene/Pd } \\
\mathrm{Se}_{2} / \text { Germaniu } \\
\mathrm{m} \\
\text { Heterojunctio } \\
\mathrm{n}^{14}\end{array}$ & $\mathbf{X}$ & प & & $\begin{array}{c}200 \sim 3000 \\
\mathrm{~nm}\end{array}$ & YES & $\begin{array}{l}\mathrm{N} / \\
\mathrm{A}\end{array}$ & $\mathbf{X}$ \\
\hline This work & Q & Q & $\begin{array}{c}\text { SPRs } \\
\text { enhanced } \\
\text { Photocurrent } \\
\text { (Fig.S14d) }\end{array}$ & $\sim 1550 \mathrm{~nm}$ & YES & $\begin{array}{l}\sim 2 \mu \mathrm{m} \\
* 4\end{array}$ & $\square$ \\
\hline
\end{tabular}

Table S1. The key performance of this work compared with previous studies. 


\section{REFERENCES}

(1) Yuan, H.; Wang, X.; Lian, B.; Zhang, H.; Fang, X.; Shen, B.; Xu, G.; Xu, Y.; Zhang, S.

C.; Hwang, H. Y.; Cui, Y., Generation and Electric Control of Spin-Valley-Coupled Circular Photogalvanic Current in $\mathrm{WSe}_{2}$. Nat. Nanotechnol. 2014, 9, 851-857.

(2) Cha, S.; Noh, M.; Kim, J.; Son, J.; Bae, H.; Lee, D.; Kim, H.; Lee, J.; Shin, H. S.; Sim, S.; Yang, S.; Lee, S.; Shim, W.; Lee, C. H.; Jo, M. H.; Kim, J. S.; Kim, D.; Choi, H., Generation, Transport and Detection of Valley-Locked Spin Photocurrent in $\mathrm{WSe}_{2}$-Graphene$\mathrm{Bi}_{2} \mathrm{Se}_{3}$ Heterostructures. Nat. Nanotechnol. 2018, 13, 910-914.

(3) McIver, J. W.; Hsieh, D.; Steinberg, H.; Jarillo-Herrero, P.; Gedik, N., Control over Topological Insulator Photocurrents with Light Polarization. Nat. Nanotechnol. 2012, 7, 96100.

(4) Lin, Z.; Rusch, L. A.; Chen, Y.; Shi, W., Optimal Ultra-Miniature Polarimeters in Silicon Photonic Integrated Circuits. APL Photonics 2019, 4, 100806.

(5) Espinosa-Soria, A.; Rodriguez-Fortuno, F. J.; Griol, A.; Martinez, A., On-Chip Optimal Stokes Nanopolarimetry Based on Spin-Orbit Interaction of Light. Nano Lett. 2017, 17, 31393144.

(6) Arbabi, E.; Kamali, S. M.; Arbabi, A.; Faraon, A., Full-Stokes Imaging Polarimetry Using Dielectric Metasurfaces. ACS Photonics 2018, 5, 3132-3140.

(7) Rubin, N. A.; D'Aversa, G.; Chevalier, P.; Shi, Z.; Chen, W. T.; Capasso, F., Matrix Fourier Optics Enables a Compact Full-Stokes Polarization Camera. Science 2019, 365, 43.

(8) Wu, W.; Yu, Y.; Liu, W.; Zhang, X., Fully Integrated CMOS-Compatible Polarization Analyzer. Nanophotonics 2019, 8, 467-474.

(9) Lee, K.; Yun, H.; Mun, S.-E.; Lee, G.-Y.; Sung, J.; Lee, B., Ultracompact Broadband Plasmonic Polarimeter. Laser Photonics Rev. 2018, 12, 1700297.

(10) Thomaschewski, M.; Yang, Y.; Wolff, C.; Roberts, A. S.; Bozhevolnyi, S. I., On-Chip Detection of Optical Spin-Orbit Interactions in Plasmonic Nanocircuits. Nano Lett. 2019, 19, 1166-1171.

(11) Afshinmanesh, F.; White, J. S.; Cai, W.; Brongersma, M. L., Measurement of the Polarization State of Light Using an Integrated Plasmonic Polarimeter. Nanophotonics 2012, 1, 125-129.

(12) Li, W.; Coppens, Z. J.; Besteiro, L. V.; Wang, W.; Govorov, A. O.; Valentine, J., Circularly Polarized Light Detection with Hot Electrons in Chiral Plasmonic Metamaterials. Nat. Commun. 2015, 6, 8379.

(13) Yuan, H.; Liu, X.; Afshinmanesh, F.; Li, W.; Xu, G.; Sun, J.; Lian, B.; Curto, A. G.; Ye, G.; Hikita, Y.; Shen, Z.; Zhang, S. C.; Chen, X.; Brongersma, M.; Hwang, H. Y.; Cui, Y., Polarization-Sensitive Broadband Photodetector Using a Black Phosphorus Vertical $p-n$ Junction. Nat. Nanotechnol. 2015, 10, 707-713. 
(14) Wu, D.; Guo, J.; Du, J.; Xia, C.; Zeng, L.; Tian, Y.; Shi, Z.; Tian, Y.; Li, X. J.; Tsang, Y. H.; Jie, J., Highly Polarization-Sensitive, Broadband, Self-Powered Photodetector Based on Graphene/PdSe 2 /Germanium Heterojunction. ACS Nano 2019, 13, 9907-9917. 